\title{
Voice disorders in severe obstructive sleep apnea patients and comparison of two acoustic analysis software programs: MDVP and Praat
}

\author{
Mei Wei ${ }^{1,2,3,4,5}$. Jianqun Du ${ }^{1,2,3,4,5} \cdot$ Xiaoyu Wang ${ }^{1,2,3,4,5} \cdot$ Honghua Lu $^{1,2,3,4,5} \cdot$ Wei Wang ${ }^{1,2,3,4,5} \cdot$ Peng Lin ${ }^{1,2,3,4,5}$
}

Received: 16 October 2019 /Revised: 11 April 2020 / Accepted: 5 May 2020 / Published online: 25 June 2020

(C) The Author(s) 2020

\begin{abstract}
Objective The purposes of this study were to explore the effect of obstructive sleep apnea-hypopnea syndrome (OSAHS) on the voice by analyzing the acoustic parameters between patients with OSAHS and those without OSAHS and to compare acoustic analyses performed by two software programs (MDVP and Praat).

Methods Patients with OSAHS $(n=75)$ and normal controls $(n=46)$ were asked to produce a sustained sound of the vowel $/ \mathrm{i} /$ and were analyzed with electroglottography (EGG), MDVP, and Praat software. A self-rated scale (Voice Handicap Index, VHI10) and acoustic parameters were compared.

Results There were no statistically significant differences in the fundamental frequency (F0), jitter, shimmer, noise/harmonic ratio (NHR), contact quotient perturbation (CQP), or contact index perturbation (CIP) between the patient group and the normal group. The VHI-10 values were significantly increased in patients with OSAHS. The receiver operating characteristic (ROC) analysis suggested that the shimmer obtained from MDVP and Praat possessed relatively high accuracy in differentiating patients with OSAHS from healthy individuals. The results for F0, jitter, shimmer, and NHR were significantly different between MDVP and Praat in OSAHS patients. In normal persons, there was a significant difference in NHR; however, no significant differences were found for F0, jitter, or shimmer between the two software programs. The results demonstrated that high correlations were found between values obtained by both software programs.

Conclusions Patients with OSAHS were prone to vibration irregularity, incomplete glottal closure, hoarseness, and other vocal problems. The two acoustic software programs present different values of acoustic measures. There was a strong correlation and consistency between the parameters calculated by the two software programs.
\end{abstract}

Keywords OSAHS $\cdot$ Acoustic analysis $\cdot$ MDVP $\cdot$ Praat $\cdot$ EGG

Wei Wang

wwei1106@hotmail.com

Peng Lin

ldusky0801@163.com

1 Department of Otorhinolaryngology Head and Neck Surgery, Tianjin First Central Hospital, No. 24 Fukang Road, Nankai District, Tianjin 300192, China

2 Institute of Otolaryngology of Tianjin, Tianjin, China

3 Key Laboratory of Auditory Speech and Balance Medicine, Tianjin, China

4 Key Clinical Discipline of Tianjin (Otolaryngology), Tianjin, China

5 Otolaryngology Clinical Quality Control Centre, Tianjin, China

\section{Introduction}

Obstructive sleep apnea-hypopnea syndrome (OSAHS) is characterized by the narrowing and collapse of pharyngeal structures during sleep, resulting in intermittent hypoxemia and sleep disruption. One or more sites of obstruction may include the soft palate, tongue, lateral walls, and/or epiglottis [1]. OSAHS has been identified as a risk factor for hypertension, heart disease, stroke, and even cancer [2-7]. This disease affects approximately 4 to $7 \%$ of adults in the general population [8]. In addition to repetitive interruptions in breathing during sleep, patients may have some signs and symptoms, including chronic snoring, memory or concentration problems, daytime sleepiness, sore throat, or dry mouth [9].

OSAHS patients have structural abnormalities in the upper airway (upper airway inflammation, thickened pharyngeal 
walls, hypertrophic tonsils, or a thickened and slack, soft palate), which lead to structural changes, and these changes affect voice production and resonance and contribute to abnormal voice features $[10,11]$. It is possible that dryness and inflammation in the upper respiratory system may be caused by chronic snoring, which may adversely affect the health of the vocal folds and lead to disturbances in phonation. Recently, a study indicated that individuals who snore for a long time showed degraded voice quality compared with the voice quality of those who do not snore [12].

In this study, we focused on acoustic analysis software to assess OSAHS patients' voice disorders by comparing the results of the acoustic analyses performed by two software programs: MDVP (Kay Elemetrics) and Praat (Boersma and Weenink). The two software programs are commonly used for acoustic analysis in clinical and research settings, while MDVP is the most used and cited acoustic analysis software [13]. Praat is distributed for free use and supported by many clinicians and scientists. The acoustic analysis software was used to objectively define F0, jitter, shimmer, and the noiseto-harmonics ratio (NHR). EGG can be used to monitor the vibrational patterns of the vocal folds. The advantage of EGG in the evaluation of the glottal wave was that it captures vibrations directly above the thyroid cartilage, without interference of the supraglottal activity or background noise. Contact index perturbation (CIP) and contact quotient perturbation (CQP) were obtained by EGG.

The aim of this study was to assess acoustic features of voice in OSAHS patients and to identify whether the results obtained from the same voice samples are comparable and/or correlative between the MDVP and Praat acoustic computer programs.

\section{Patients and methods}

\section{Patients}

This was a prospective study performed at Tianjin First Central Hospital. Two groups of subjects were recruited. The patient group consisted of 75 patients who were diagnosed with severe OSAHS (apnea-hypopnea index $>30 / \mathrm{h}$ ) [14] by polysomnographic examination. According to the imaging examination, all the patients were obstructed by the pharynx and pharyngeal obstruction. The appearance and activity of the vocal cords under stroboscopic laryngoscopy were normal. The normal group included 46 healthy people with no symptoms, such as excessive daytime sleepiness, loud snoring, observed episodes of breathing cessation during sleep, abrupt awakenings accompanied by shortness of breath, and awakening with dry mouth or sore throat. The STOPBang questionnaire was used to screen for OSAHS in the normal group. The STOP-Bang questionnaire is a validated
OSAHS screening tool. The questionnaire has 8 items, and each item is worth 1 point. If the score of the first 4 items added is $\geq 2$ points, the risk of OSAS is considered to be high; if the STOP-Bang questionnaire is used completely, and the total score is $\geq 3$, the risk of OSAS is considered to be high. The participants in the normal control group needed to complete 8 items and score no more than 3 points. The people in the two groups were male and had similar age and BMI distributions. Exclusion criteria for both the patient and normal group were smoking, comorbid diseases likely to affect the voice, using medications, history of laryngeal problems, Sjogren's syndrome, pulmonary or neurologic disease, and other autoimmune disorders likely to change voice production.

\section{Acoustic voice analysis}

Each voice sample was recorded while the examiner was under identical conditions in a sound-treated room with ambient noise below $50 \mathrm{~dB}$. The person took a seat in front of the microphone placed $15 \mathrm{~cm}$ from their mouths. To reduce intrasubject variability, 5 samples of sustained vowel /i/ sounds at a comfortable pitch, constant amplitude, and flat tone were used for each subject. All voice samples were obtained using EGG and Speech Lab Model 4500 (Kay Elemetrics Corporation) at a sampling rate of $50 \mathrm{kHz}$ and 16-bit quantization. Acoustic voice analysis was performed twice: once using MDVP and once using Praat. The fundamental frequency (F0), jitter, shimmer, and NHR were compared between MDVP and Praat. Additionally, CQP and CIP were obtained by EGG.

The Turkish version of the Voice Handicap Index-10 (VHI-10) was used to evaluate voice quality. The VHI-10 is a questionnaire that assesses a patient's perception of the physical, emotional, and functional aspects of their voice [15]. Subjects receive a score of $0-4$ for each question. The higher the score, the greater the degree of handicap.

In the present study, all acoustic voice parameters were evaluated in the morning, which prevented the voices of the participants from being affected by circadian changes [16].

\section{Statistical analysis}

Statistical analysis of the data was performed using IBM SPSS statistics 19. The Mann-Whitney $U$ test was used to compare the four markers between normal persons and patients. The receiver operating characteristics (ROC) approach was also applied to evaluate the diagnostic value of voice parameters in OSAHS patients, and the results were estimated by calculating the area under the ROC curve (AUC), sensitivity, and specificity according to standard formulas. Spearman's rank correlation coefficient (Spearman's rho) and Bland-Altman analysis were performed to assess the correlation and 
Table 1 The results of VHI-10 and comparison of acoustic parameters between OSAHS patients and normal persons

\begin{tabular}{llccl}
\hline Parameter & Software & OSAHS & Normal & $\begin{array}{l}\text { Cohen's } \\
d\end{array}$ \\
& & & & \\
\hline F0 & MDVP & $130.774 \pm 26.367$ & $139.812 \pm 26.713$ & 0.268 \\
& Praat & $128.455 \pm 25.498$ & $135.553 \pm 23.468$ & 0.338 \\
Jitter & MDVP* & $1.489 \pm 0.822$ & $0.541 \pm 0.352$ & 1.383 \\
& Praat* & $0.945 \pm 0.602$ & $0.522 \pm 0.267$ & 0.840 \\
Shimmer & MDVP* & $4.189 \pm 1.571$ & $2.683 \pm 0.624$ & 1.159 \\
& Praat* & $5.074 \pm 1.96$ & $2.860 \pm 0.792$ & 1.358 \\
NHR & MDVP* & $0.147 \pm 0.029$ & $0.123 \pm 0.016$ & 0.975 \\
& Praat* & $0.047 \pm 0.259$ & $0.037 \pm 0.052$ & 0.257 \\
VHI* & & $3.09 \pm 2.98$ & $1.87 \pm 2.08$ & 0.453 \\
CQP* & EGG & $6.12 \pm 6.11$ & $2.19 \pm 2.14$ & 0.778 \\
CIP* & EGG & $42.83 \pm 47.82$ & $17.52 \pm 13.80$ & 0.272 \\
\hline
\end{tabular}

*Indicates a significant difference

consistency of F0, jitter, shimmer, and NHR between the two acoustic voice programs: MDVP and Praat.

\section{Results}

\section{Acoustic characteristics of OSAHS patients}

The results of the acoustic analysis performed using the two software programs in normal persons and OSAHS patients are shown in Table 1. Statistical analysis revealed that there was no statistically significant difference between the normal group and the patient group in F0 according to the two analysis programs.

In the MDVP analysis, there were significant differences in jitter, shimmer, and NHR between the two groups. The ROC curve analysis is shown in Fig. 1a. The AUC of jitter was 0.840 (95\% CI 0.767-0.914), and the cutoff point was

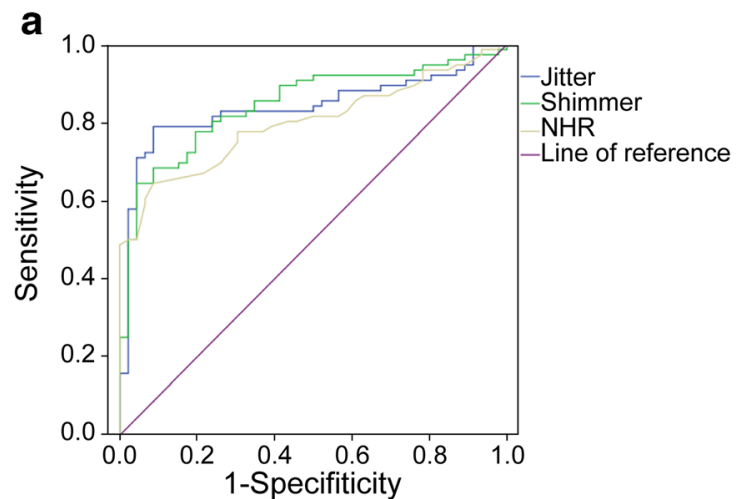

0.774 , with a sensitivity of $78.9 \%$ and specificity of $91.3 \%$. The AUC of shimmer was 0.847 (95\% CI 0.777-0.916), and the cutoff point was 3.598 , with a sensitivity of $64.5 \%$ and specificity of $95.7 \%$. The AUC of NHR was 0.799 (95\% CI $0.723-0.876$ ), and the cutoff value was 0.141 , with a sensitivity of $65.5 \%$ and specificity of $91.3 \%$. The results showed that shimmer assessed by MDVP possessed relatively high accuracy in differentiating OSAHS patients from healthy individuals.

For Praat, significant differences were also found in jitter, shimmer, and NHR between the normal group and patient group. The ROC curve analysis showed that the AUC of jitter was 0.736 (95\% CI 0.648-0.825), and the cutoff was 0.568 , with a sensitivity of $71.05 \%$ and specificity of $71.7 \%$. The AUC of shimmer was 0.869 (0.804-0.934), and the cutoff was 3.47 , with a sensitivity of $81.5 \%$ and specificity of $80.1 \%$. Shimmer possessed relatively high accuracy in differentiating OSAHS patients from healthy individuals. The AUC of NHR was $0.689(0.590-0.789)$, and the cutoff value was 0.035 , with a sensitivity of $73.6 \%$ and specificity of $63.0 \%$ (Fig. 1b).

VHI-10 scores and acoustic parameters from EGG are also depicted in Table 1. The mean VHI-10 score of OSAHS patients was significantly higher than that of normal persons. In the OSAHS group, CQP and CIP for the EGG signals showed significantly greater values than those in the normal group $(P<0.001)$.

\section{The differences and correlations between MDVP and Praat}

The results of the acoustic analysis performed using the two software programs for the two study groups are presented in Table 2. In addition to the shimmer, the values obtained for F0, jitter, and NHR were higher for MDVP than those obtained using Praat.

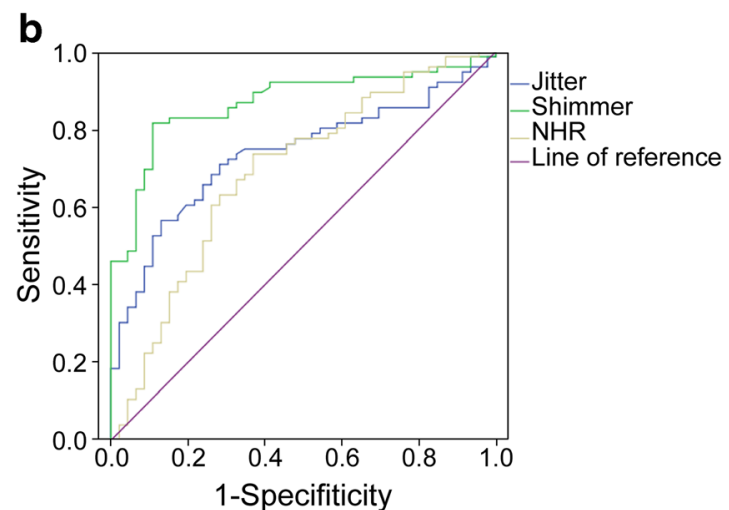

Fig. 1 a ROC curves were established to evaluate the clinical value of jitter, shimmer, and NHR from MDVP in OSAHS patients. b ROC curves were established to evaluate the clinical value of jitter, shimmer, and NHR from Praat in OSAHS patients 
Table 2 Comparison of acoustic parameters obtained by MDVP and Praat

\begin{tabular}{llccc}
\hline Parameter & Software & MDVP & Praat & Cohen's $d$ \\
\hline F0 & OSAHS $^{\#}$ & $130.774 \pm 26.367$ & $128.455 \pm 25.498$ & 0.169 \\
\multirow{2}{*}{ Jitter } & Normal & $139.812 \pm 26.713$ & $135.553 \pm 23.468$ & 0.184 \\
& OSAHS $^{\#}$ & $1.489 \pm 0.822$ & $0.945 \pm 0.602$ & 0.754 \\
\multirow{2}{*}{ Shimmer } & Normal & $0.541 \pm 0.352$ & $0.522 \pm 0.267$ & 0.062 \\
\multirow{2}{*}{ NHR } & OSAHS $^{\#}$ & $4.189 \pm 1.571$ & $5.074 \pm 1.96$ & 0.498 \\
& Normal $^{*}$ & $2.683 \pm 0.624$ & $2.860 \pm 0.792$ & 0.255 \\
& OSAHS $^{\#}$ & $0.147 \pm 0.029$ & $0.047 \pm 0.259$ & 3.653 \\
\hline
\end{tabular}

**The differences between the two programs in normal voice $P<0.05$. ${ }^{\#}$ The differences between the two programs in OSAHS patients $P<0.05$
In the normal group, statistical analysis revealed a significant difference between the results obtained from the two software programs for NHR. No significant differences were found between the two programs for the F0, jitter, or shimmer (Table 2). However, F0 determined with MDVP showed a strong correction with that determined with Praat. The MDVP jitter data also showed a strong

a

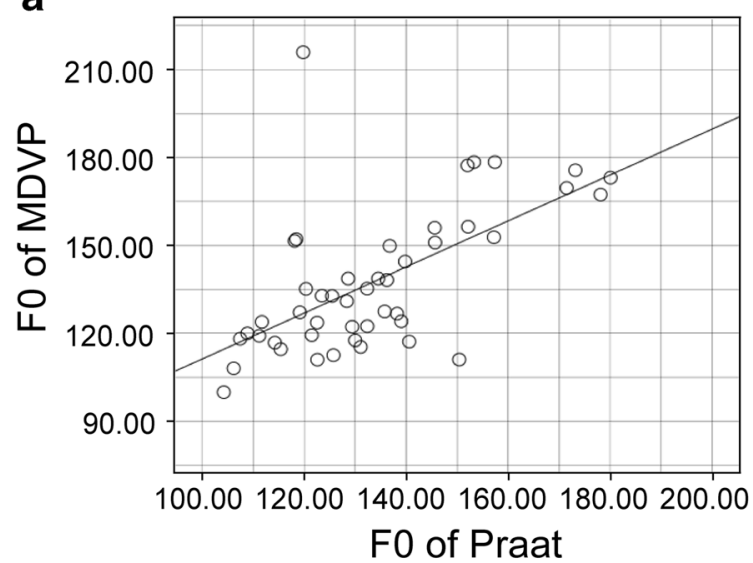

C

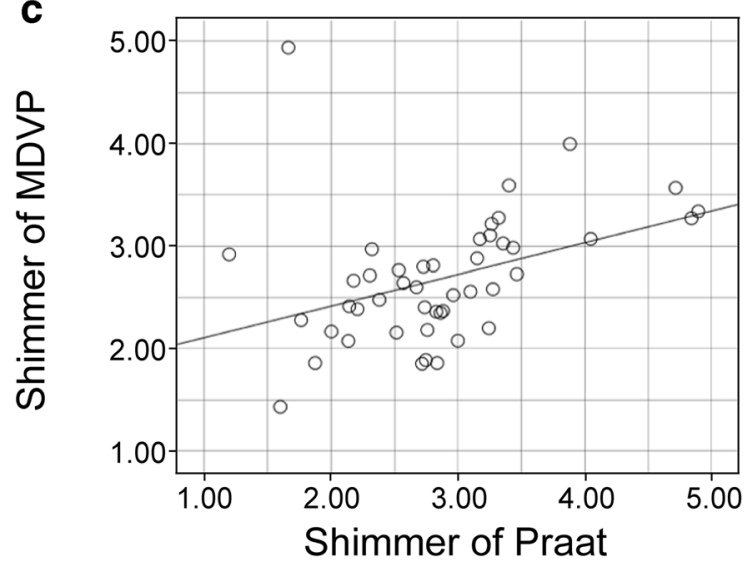

Fig. 2 Normal voice data. The horizontal axis indicates the Praat data. The vertical axis indicates the multidimensional voice program (MDVP). a Scatter plot of the fundamental frequency. Spearman's rho $=0.604$. b correlation with Praat. There was a strong correlation between MDVP and Praat regarding the NHR. Additionally, the shimmer data from Praat showed a strong correlation with MDVP (Fig. 2).

In the OSAHS patient group, there were significant differences between the results obtained from the two software programs for F0, jitter, shimmer, and NHR. The F0, jitter,

b
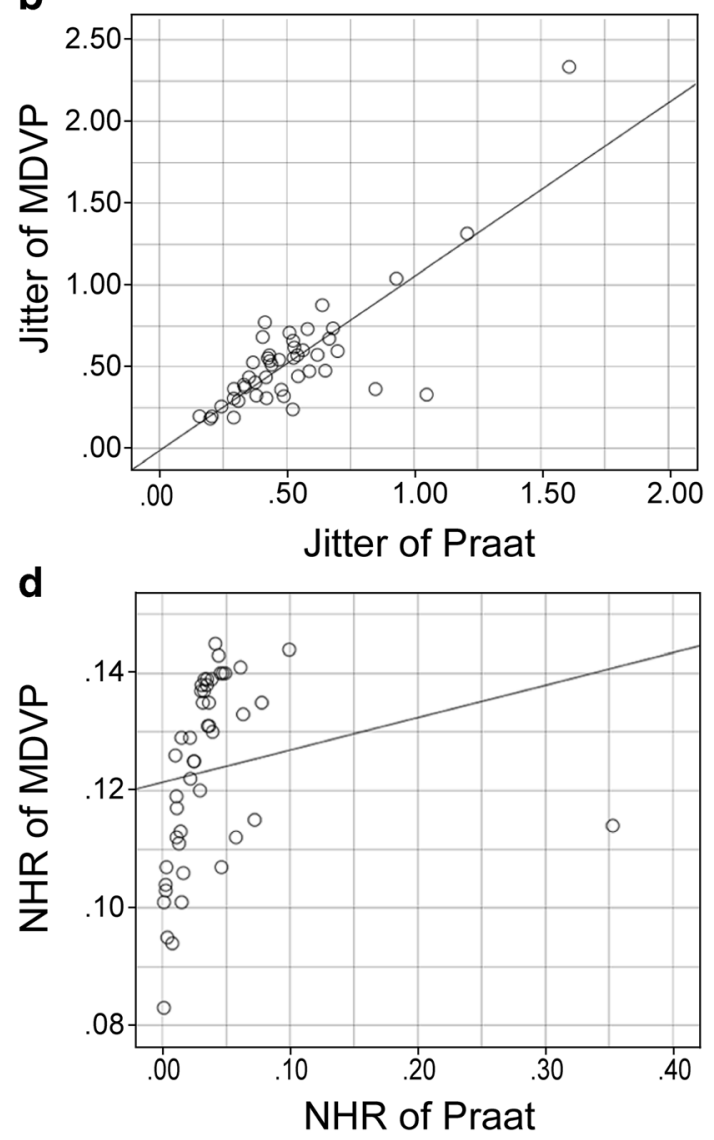

Scatter plot of jitter. Spearman's rho $=0.657$. c Scatter plot of shimmer. Spearman's rho $=0.525$. d Scatter plot of NHR. Spearman's rho $=0.665$ 

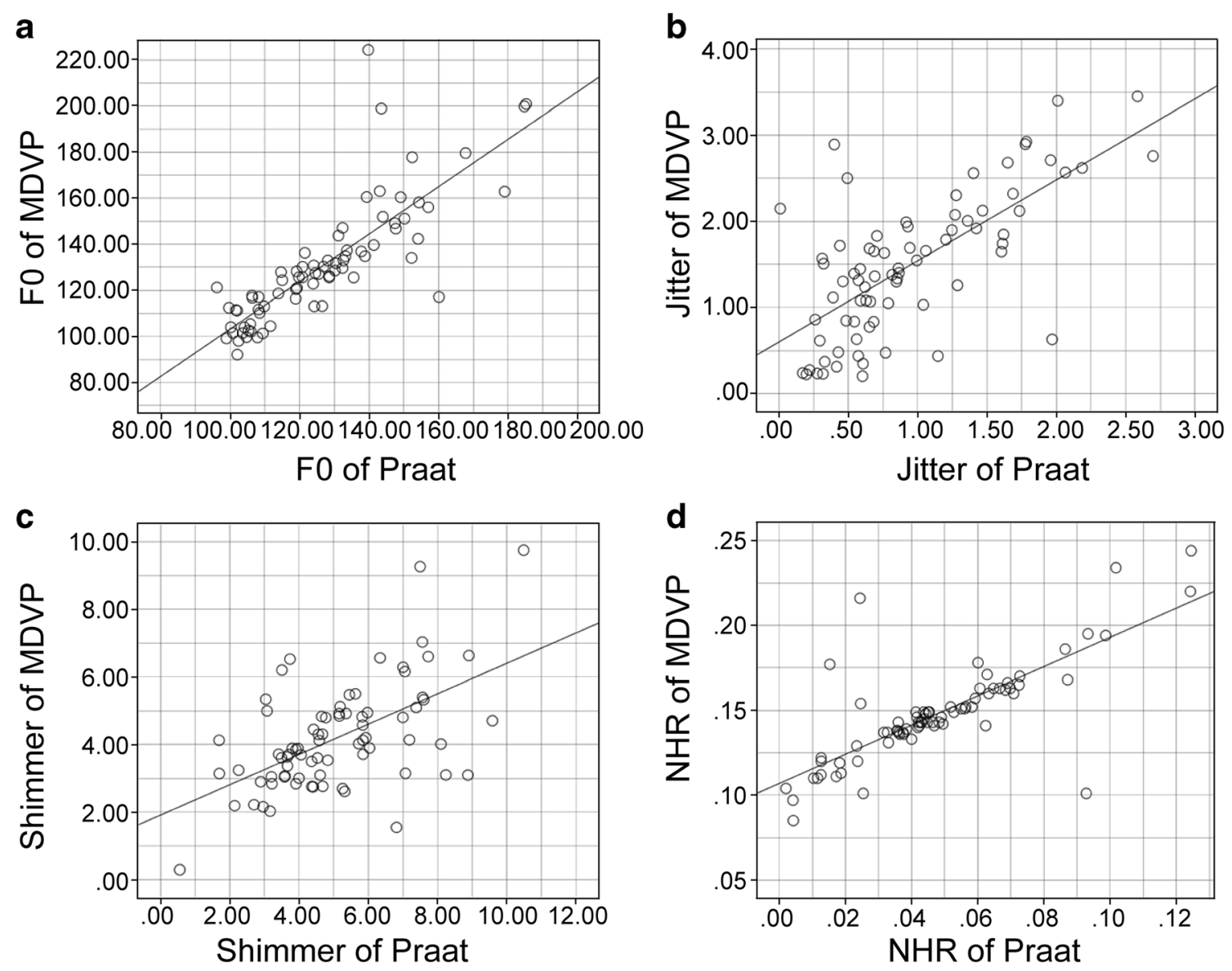

Fig. 3 Patient voice data. The horizontal axis indicates the Praat data. The vertical axis indicates the multidimensional voice program (MDVP). a Scatter plot of the fundamental frequency. Spearman's rho $=0.885$. b

shimmer, and NHR determined with MDVP showed a strong correlation with those determined with Praat (Fig. 3).

\section{Consistency of MDVP and Praat measurement parameters}

The results of the MDVP and Praat consistency tests are shown in Fig. 4. From the figure, we concluded that there were $4.10 \%$ (5/122), $3.28 \%$ (4/122), $9.01 \%$ (11/122), and $1.63 \%$ $(2 / 122)$ points outside the $95 \%$ limits of agreement (LOA) for F0, jitter, shimmer, and NHR, respectively, measured by MDVP and Praat. The 95\% LoAs of F0, jitter, shimmer, and NHR are $-29.1 \sim 37.2,-0.73 \sim 1.42,-3.5 \sim 2.2$, and $0.03 \sim$ 0.16 , respectively. Within the $95 \%$ limits of agreement, the absolute values of the difference between the two instruments are $37.215,1.423,3.476$, and 0.165 , respectively. The average of the differences are $4.0,0.35,-0.6$, and 0.09 , respectively. From a clinical perspective, this difference is acceptable, so the two approaches are consistent.

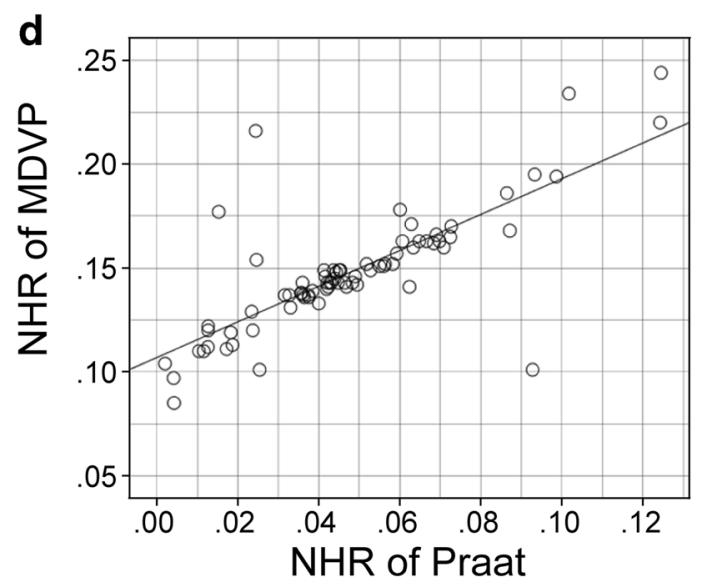

Scatter plot of jitter. Spearman's rho $=0.690$. c Scatter plot of shimmer. Spearman's rho $=0.562$. d Scatter plot of NHR. Spearman's rho $=0.772$

\section{Discussion}

It is known that the upper airway structure plays an important role in voice resonance and articulation [17]. Obstruction of any locations of the upper respiratory tract (URT) may affect the resonance and acoustic characteristics of the voice. Subramaniam et al. reported that patients with adenotonsillar hypertrophy showed significant changes in acoustic parameters compared to healthy controls [18]. On the other hand, OSAHS patients have a higher incidence of mouth breathing. Long-term mouth opening of OSAHS patients causes the inhaled airflow to be lost by the nasal cavity, causing the vocal cord surface to dry [19]. Tao et al. [20] found that with the decrease in moisture in the liquid layer on the surface of the vocal cords, the threshold voltage of the vocalization rises, and when the pressure increases to a certain extent, the vocal cords may be damaged.

This study shows that the vocal cord vibration of OSAHS patients is irregular, and the fundamental frequency perturbation, amplitude perturbation, and vibration-related acoustic parameters, such as jitter, shimmer, NHR, CQP, and CIP, 
a

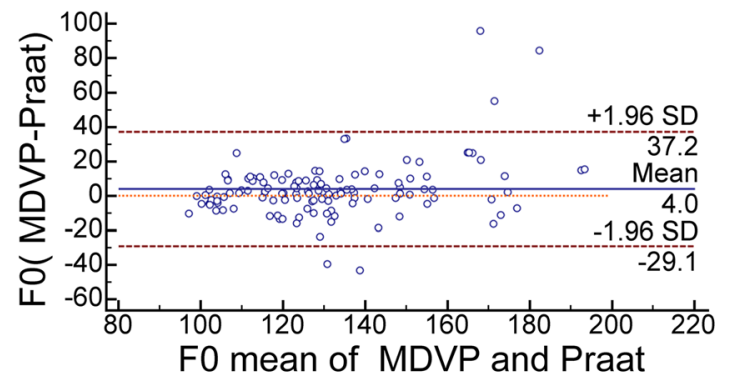

C

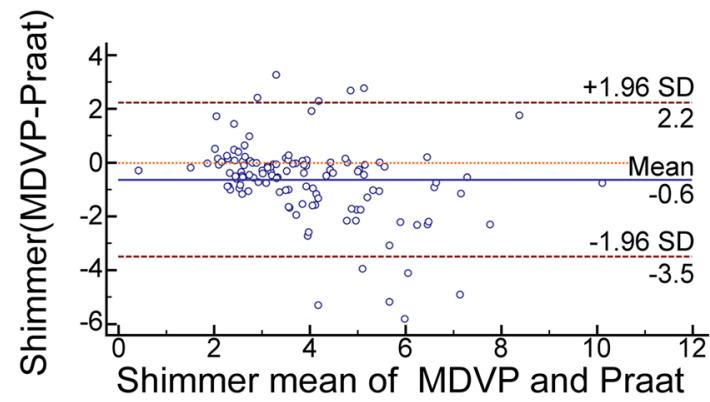

Fig. 4 Analysis of consistency between MDVP and Praat measurements. a MDVP and Praat measurements of F0. b MDVP and Praat b

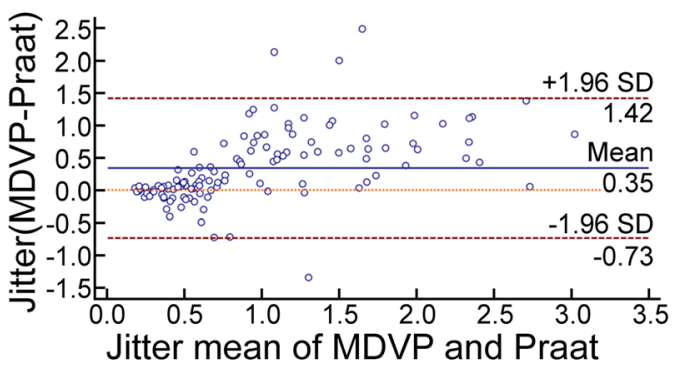

d

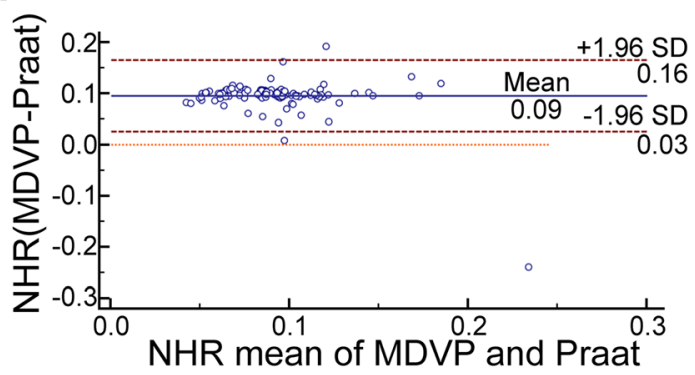

measurements of jitter. c MDVP and Praat measurements of shimmer. d MDVP and Praat measurements of NHR

the values from MDVP can be linearly transformed to approximate the value calculated by Praat.

In conclusion, OSAHS patients experience long-term mouth breathing and changes in the structure of the articulation area, which result in irregular vocal cord vibration, poor glottis closure, pronunciation difficulties, hoarseness, and other vocal problems. Therefore, in clinical treatment, attention should be paid to the patient's voice changes, and mouth-breathing habits should be corrected to avoid changes in vocal cord tissue biomechanics and vocalization due to vocal cord dehydration. The two acoustic software programs present different values of acoustic measures, and MDVP appeared to differentiate the OSAHS group better than Praat. In addition, there is a strong correlation and consistency between the values calculated by MDVP and Praat. In some cases, either of the two software programs can be used to analyze voice data.

Funding information This study was supported by the Key Clinical Discipline of Tianjin, National Natural Science Foundation of China (81971698), and Tianjin Natural Science Foundation (19JCYBJC27200).

\section{Compliance with ethical standards}

Conflict of interest The authors declare that there are no conflicts of interest.

Open Access This article is licensed under a Creative Commons Attribution 4.0 International License, which permits use, sharing, adaptation, distribution and reproduction in any medium or format, as long as you give appropriate credit to the original author(s) and the source, provide a link to the Creative Commons licence, and indicate if changes were made. The images or other third party material in this article are included 
in the article's Creative Commons licence, unless indicated otherwise in a credit line to the material. If material is not included in the article's Creative Commons licence and your intended use is not permitted by statutory regulation or exceeds the permitted use, you will need to obtain permission directly from the copyright holder. To view a copy of this licence, visit http://creativecommons.org/licenses/by/4.0/.

\section{References}

1. Kezirian EJ, Hohenhorst W, de Vries N (2011) Drug-induced sleep endoscopy: the VOTE classification. Eur Arch Otorhinolaryngol 268(8):1233-1236

2. Tregear S, Reston J, Schoelles K, Phillips B (2010) Continuous positive airway pressure reduces risk of motor vehicle crash among drivers with obstructive sleep apnea: systematic review and metaanalysis. Sleep 33(10):1373-1380

3. Yaggi HK, Concato J, Kernan WN, Lichtman JH, Brass LM, Mohsenin V (2005) Obstructive sleep apnea as a risk factor for stroke and death. N Engl J Med 353(19):2034-2041

4. Martinez D, Klein C, Rahmeier L, da Silva RP, Fiori CZ, Cassol CM, Gonçalves SC, Bos AJG (2012) Sleep apnea is a stronger predictor for coronary heart disease than traditional risk factors. Sleep Breath 16(3):695-701

5. Marin JM, Agusti A, Villar I, Forner M, Nieto D, Carrizo SJ, Barbé F, Vicente E, Wei Y, Nieto FJ, Jelic S (2012) Association between treated and untreated obstructive sleep apnea and risk of hypertension. Jama 307(20):2169-2176

6. Marshall NS, Wong KK, Cullen SR et al (2014) Sleep apnea and 20-year follow-up for all-cause mortality, stroke, and cancer incidence and mortality in the Busselton Health Study cohort. J Clin Sleep Med 10(4):355-362

7. Young T, Finn L, Peppard PE et al (2008) Sleep disordered breathing and mortality: eighteen-year follow-up of the Wisconsin sleep cohort. Sleep 31(8): 1071-1078

8. Lim JS, Lee JW, Han C, Kwon JW (2018) Correlation of soft palate length with velum obstruction and severity of obstructive sleep apnea syndrome. Auris Nasus Larynx 45(3):499-503

9. Lurie A (2011) Obstructive sleep apnea in adults: epidemiology, clinical presentation, and treatment options. Adv Cardiol 46:1-42

10. Han D, Xu W, Hu R, Zhang L (2012) Voice function following Han's uvulopalatopharyngoplasty. J Laryngol Otol 126(1):47-51
11. Espinoza-Cuadros F, Fernandez-Pozo R, Toledano DT et al (2015) Speech signal and facial image processing for obstructive sleep apnea assessment. Comput Math Methods Med 2015:489761

12. Hamdan AL, Al-Barazi R, Kanaan A et al (2012) The effect of snoring on voice: a controlled study of 30 subjects. Ear Nose Throat J 91(1):28-33

13. Smits I, Ceuppens P, De Bodt MS (2005) A comparative study of acoustic voice measurements by means of Dr. Speech and Computerized Speech Lab. J Voice 19(2):187-196

14. Myers KA, Mrkobrada M, Simel DL (2013) Does this patient have obstructive sleep apnea?: the rational clinical examination systematic review. Jama 310(7):731-741

15. Rosen CA, Lee AS, Osborne J, Zullo T, Murry T (2004) Development and validation of the voice handicap index-10. Laryngoscope 114(9):1549-1556

16. Morton LL, Diubaldo D (1993) Circadian differences in the dichotic processing of voicing. Int J Neurosci 68(1-2):43-52

17. Bertino G, Matti E, Migliazzi S, Pagella F, Tinelli C, Benazzo M (2006) Acoustic changes in voice after surgery for snoring: preliminary results. Acta Otorhinolaryngol Ital 26(2):110-114

18. Subramaniam V, Kumar P (2009) Impact of tonsillectomy with or without adenoidectomy on the acoustic parameters of the voice: a comparative study. Arch Otolaryngol Head Neck Surg 135(10): 966-969

19. Titze IR (1992) Phonation threshold pressure: a missing link in glottal aerodynamics. J Acoust Soc Am 91(5):2926-2935

20. Tao C, Jiang JJ, Zhang Y (2009) A fluid-saturated poroelastic model of the vocal folds with hydrated tissue. J Biomech 42(6):774-780

21. Deliyski DD, Shaw HS, Evans MK, Vesselinov R (2006) Regression tree approach to studying factors influencing acoustic voice analysis. Folia Phoniatr Logop 58(4):274-288

22. Amir O, Wolf M, Amir N (2009) A clinical comparison between two acoustic analysis softwares: MDVP and Praat. Biomedical Signal Processing and Control 4(3):202-205

23. Vasilakis M, Stylianou Y (2009) Spectral jitter modeling and estimation[J]. Biomed Signal Proces 4(3):183-193

24. Oguz H, Kilic MA, Safak MA (2011) Comparison of results in two acoustic analysis programs: Praat and MDVP[J]. Turk J Med Sci 41(5):835-841

Publisher's note Springer Nature remains neutral with regard to jurisdictional claims in published maps and institutional affiliations. 